\title{
BMJ Open Quality Shoulder Reduction Bench Project: improving care for patients with shoulder dislocations
}

Philip Dowson

To cite: Dowson P. Shoulder Reduction Bench Project: improving care for patients with shoulder dislocations. BMJ Open Quality 2019;8:e000366. doi:10.1136/ bmjoq-2018-000366

Received 14 March 2018 Revised 23 April 2019 Accepted 6 May 2019
Check for updates

(C) Author(s) (or their employer(s)) 2019. Re-use permitted under CC BY-NC. No commercial re-use. See rights and permissions. Published by BMJ.

Emergency Department, City Hospitals Sunderland NHS Foundation Trust, Sunderland, UK

Correspondence to

Dr Philip Dowson;

phildowson@doctors.org.uk

\section{ABSTRACT}

This paper presents the background, methodology and results of a quality improvement project undertaken at a district general hospital. The project was launched in response to the concerning results from audit data which showed significant delays in the treatment of patients with shoulder dislocations and a high percentage of patients receiving procedural sedation. Using 'Plan-Do-Study-Act' cycles involving training sessions, written protocols and an online video, we were able to train a large cohort of nurse practitioners in the use of the Shoulder Reduction Bench. This is a relatively novel, evidence-based technique for reducing shoulder dislocations without the need for sedation. The new shoulder dislocation protocol was successful in reducing the average time from presentation to shoulder relocation by $31 \mathrm{~min}$ and the average time from presentation to discharge by $52 \mathrm{~min}$. It also resulted in a $68 \%$ reduction in the number of patients receiving procedural sedation over a 6-month period. This project inspired the practitioners, most of whom had never reduced a shoulder dislocation before. The success of the new shoulder reduction bench protocol prompted interest from the trust's innovation department and has been publicised both within the trust and regionally. This publicity and the satisfaction gained by the staff from this effective new skill have helped to anchor the change in departmental culture. Link to training video: https://www. youtube.com/watch?v=40aCqhfQXD4\&feature=youtu.be

\section{INTRODUCTION}

Shoulder dislocation is the most common large joint dislocation seen in the emergency department with an incidence in the UK of 40.4 per 100000 person-years in men and 15.5 per 100000 person-years in women..$^{1}$ It is an extremely painful and debilitating injury.

The ability to treat a shoulder dislocation is vital for emergency medicine practitioners, and a department should strive to treat them as efficiently as possible.

\section{Problem description}

In September 2016, a departmental audit had highlighted poor compliance with the Royal College of Emergency Medicine guidelines on the time taken to reduce shoulder dislocations. ${ }^{2}$ A sample of 30 patients with a coded diagnosis of shoulder dislocation from September 2015 to September 2016 was analysed. The inclusion criteria were patients with confirmed shoulder dislocations on $\mathrm{X}$-ray, who were successfully treated and discharged from the emergency department. Since the paediatric department is managed as a separate unit, we excluded patients under 16 years old.

The key outcomes were measured, and the results were as follows:

- 2 hours 25 min average from triage to confirmed shoulder reduction X-ray (range: 1 hour $14 \mathrm{~min}$ to 5 hours $3 \mathrm{~min}$ ),

- 3 hours 22 min average triage to discharge (range: 1 hour $46 \mathrm{~min}$ to 6 hours $39 \mathrm{~min}$ ), - 57\% (17/30) patients sedated for shoulder reduction.

These results were striking as shoulder dislocations can and should be managed promptly. We also found a sedation rate of $57 \%$ concerning. Previous literature has quoted a $4 \%$ risk of an adverse event following procedural sedation in the emergency department. ${ }^{3}{ }^{4}$ National guidance mandates a minimum of two physicians (one performing the procedure, one administering sedation) and one nurse to safely sedate a patient. ${ }^{5}$

Reducing shoulder dislocations can be extremely uncomfortable for the patient, and associated muscle spasm can be difficult for the physician to overcome. To facilitate the reduction, an array of different medication strategies can be used. For our project, we defined analgesia as the use of opioid analgesia (ie, Morphine) with or without an analgesic with anxiolytic properties (ie, nitrous oxide). Sedation is defined as a drug-induced depression of consciousness, typically provided in our department with a benzodiazepine (Midazolam) and/or a general anaesthetic agent (Propofol).

Quality of patient care can be defined as a service that is safe, caring, responsive, efficient and well-led. ${ }^{6}$ When applying this definition to the identified problem, the initial questions were addressed as given in table 1 . 
Table 1 Initial project planning questions

$\begin{array}{ll}\text { Safe } & \text { Do we need to be sedating as many patients as we do? } \\ \text { Caring } & \text { How can we provide these patients with more prompt shoulder reductions? } \\ \text { Responsive } & \text { How do we analyse and respond to the department's poor performance? } \\ \text { Efficient } & \text { How can we streamline the process from triage to successful treatment for patients? } \\ \text { Well-led } & \begin{array}{l}\text { Can we lead the whole team through a change in culture surrounding the } \\ \text { management of shoulder dislocations? }\end{array}\end{array}$

\section{Rationale}

Through process mapping, we identified that a patient presenting to our emergency department with a shoulder dislocation will typically be managed along one of three distinct pathways (figures 1 and 2). All patients present to triage and wait to be seen by their initial practitioner which may be an emergency nurse practitioner (ENP) or a doctor. The patient is then sent for X-ray to confirm the diagnosis of a shoulder dislocation. Fifty-seven per cent of patients were then transferred to a monitored bed to receive procedural sedation prior to treatment. These patients then wait to recover from sedation before receiving a further X-ray to confirm successful joint reduction prior to discharge. In $27 \%$ of cases, the initial practitioner would hand the patient over to a senior doctor who would perform the shoulder reduction with analgesia only. Only in the remaining $16 \%$ of cases would the initial practitioner treat the patient autonomously with analgesia only. The process map (figure 1) demonstrates the additional monitoring, staffing and potential delays caused by a patient requiring procedural sedation. These delays are avoided by the initial practitioner reducing the shoulder with analgesia only where possible.

Having analysed the data, we needed to change the current departmental culture and encourage more reductions with analgesia only by the initial practitioner assessing the patient. The majority of our shoulder dislocations were initially assessed by ENPs (53\%) who then waited until a senior doctor was available to handover their care once a dislocation was confirmed. Only one in four of those practitioners took any further role in that patient's reduction or management. For this project to

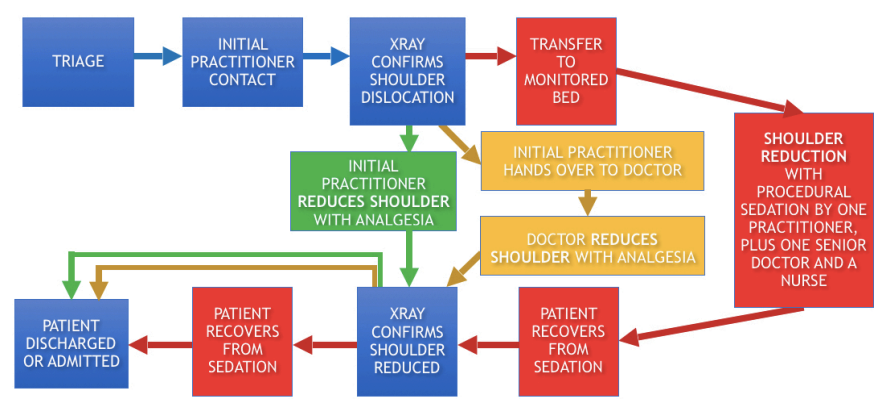

Figure 1 Process map identifying the steps that each patient presenting with a shoulder dislocation may take before they can be discharged from the emergency department. Each arrow represents a potential delay for that patient. be successful, we needed to engage and 'up-skill' the ENPs. To do this required a protocol and recommended technique that was effective and easy to teach. The Royal College of Emergency Medicine guidelines are consensus based and do not refer to any particular method of shoulder reduction; this is due to a lack of convincing evidence regarding the optimum method of shoulder reduction.

\section{Available knowledge}

We were aware from recent literature of the Oxford Chair technique. Initially devised by Dr Phil Hormbrey of Oxford University Hospitals, this equipment and technique has been used as their primary reduction method for shoulder dislocations for the past 12 years. It was originally reported from a retrospective service evaluation to have a success rate of $62 \%$ without sedation. ${ }^{7}$ The theory is that the chair positioning enables the practitioner to recreate the position at which the shoulder joint is the most unstable and uses gravity as the main counter force. ${ }^{8}$ This means fewer leverage forces; these have previously been documented as giving rise to a $1 \%$ risk of further injury. ${ }^{9}$ Its effectiveness has been recently demonstrated in a randomised control trial by Chung et al. ${ }^{10}$ This trial demonstrated a $77 \%$ reduction success rate with no sedation required and had reduced the median length of stay in the emergency department by $21 \mathrm{~min}(\mathrm{p}=0.183)$. Although a small study of 60 patients with poor statistical significance when considering the length of stay, we were struck by the success rate of the technique.

Table 2 shows the literature search strategy undertaken to identify any other literature pertaining to the Oxford Chair.

While not assessing the Oxford Chair specifically, a study by Guler et al performed a comparative retrospective analysis comparing the 'Chair' method which is similar in its approach using a standard chair backrest as a fulcrum. This study found that physicians felt the

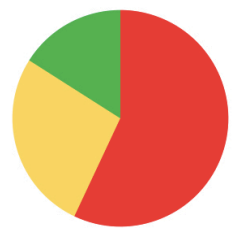

RED: SEDATION ROUTE $57 \%$
AMBER: HAND OVER ANALGESIA ONLY ROUTE $27 \%$
GREEN: SINGLE PRACTITIONER ANALGESIA ONLY ROUTE $16 \%$

Figure 2 Pie chart based on initial audit data showing what percentage of patients with shoulder dislocations are managed along each process pathway. 
Table 2 Literature search strategy

\begin{tabular}{|c|c|}
\hline Databases searched & EMBASE, Medline, PubMed, BNI, CINAHL \\
\hline Search terms & $\begin{array}{l}\text { (((shoulder).ti,ab OR (glenohumeral).ti,ab) AND (chair).ti,ab) AND } \\
\text { exp DISLOCATION/ }\end{array}$ \\
\hline No. of papers identified & 370 \\
\hline Papers referring to Oxford Chair or similar techniques & 9 \\
\hline
\end{tabular}

technique was easier to perform and had a significantly better reduction time $(3.0 \pm 1.2 \mathrm{~min}, \mathrm{p}=0.011)$ and visual analogue pain scores $(4.0 \pm 2.4, \mathrm{p}=0.03)$ when compared with three other traditional methods. This Turkish study also highlighted clear cultural differences between our practice and what is accepted internationally, as all the patients in their study had their shoulders successfully reduced without any form of sedation. While this may not be generalisable due to patient expectations of pain management in the UK, it appeared to support feelings that our sedation rate was higher than necessary.

Having requested the plans for the Oxford Chair from Dr Hormbrey, the design was modified, made adjustable and then manufactured as the 'Shoulder Reduction Bench' (figure 3). As a non-sterile piece of equipment without a measuring function, the bench was deemed by our Innovations Department to be a Class 1, low risk, medical device. These do not require involvement of a notified body. According to the Medicines and Healthcare Products Regulatory Agency (MHRA) guidance, as the bench was manufactured 'in-house' for the use of the patients within our institution with no current plans to place it on the market, no conformité européenne (CE) marking is required. ${ }^{12}$ Attempts are underway with local design companies to create a more durable prototype for use in the years to come. The author has no financial interest in the bench.

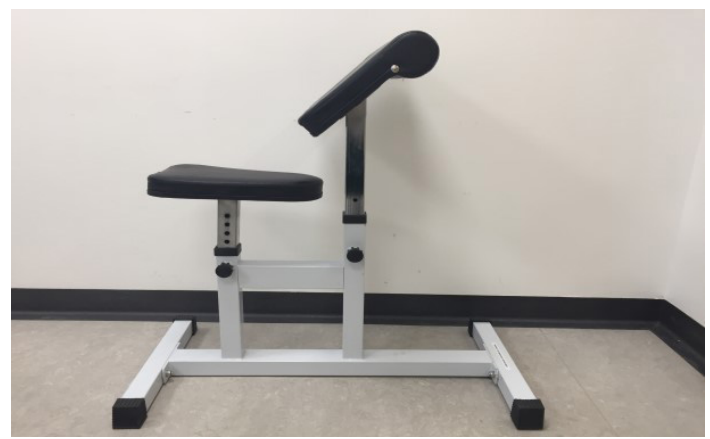

Figure 3 The modified Shoulder Reduction Bench with adjustable chest rest.
Specific aims

The proposed aims of our project were as follows:

What are we trying to achieve?

Improve the experience and safety of patients presenting with shoulder dislocations by providing more prompt treatment and less exposure to procedural sedation.

How will we know that a change is an improvement?

Reduced time to shoulder reduction would indicate that patients are receiving more rapid treatment of a severely painful injury. Reduced time to discharge would indicate that patients are being subjected to fewer delays before being able to go home. Reduced number of patients receiving procedural sedation would indicate fewer patients being exposed to the potential risk of adverse events from potent anaesthetic drugs.

What changes can we make that will result in an improvement? Provide the equipment and training to the emergency department nursing and medical practitioners to enable them to independently perform a simple method of shoulder reduction that is proven to have a high success rate without the need for procedural sedation.

\section{METHODS}

\section{Context and interventions}

Having acquired agreement for the project from the Clinical Governance Group, we then formed a guiding coalition. The key player groups highlighted in the stakeholder analysis matrix (figure 4) needed to be included in decision making and be regularly consulted. As such the proposed guiding coalition was as follows: an emergency medicine consultant, a junior doctor and an ENP. Including an ENP in the guiding coalition carried the added benefit of them being a permanent staff member, as opposed to the frequently rotating junior doctors. This would be of importance when it came to anchoring the change in the departmental culture, as permanent staff members' involvement would help ensure our message did not get lost when other staff members are regularly replaced.

The initial training session took place as part of the ENPs' monthly departmental teaching. Some nurse practitioners had previously described a reluctance to engage in certain procedures, including shoulder reductions. This was due to the perceived risks involved and the feeling that nurse practitioners are more subject to 


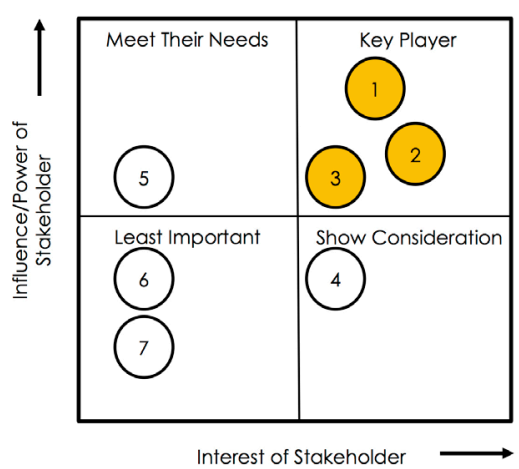

$$
\begin{aligned}
& \text { 1. Emergency Medicine } \\
& \text { Consultants } \\
& \text { 2. Emergency Medicine Junior } \\
& \text { Doctors } \\
& \text { 3. Emergency Nurse Practitioners } \\
& \text { 4. Emergency Department Nursing } \\
& \text { Staff } \\
& \text { 5. Trauma and Orthopaedic } \\
& \text { 6. Ponsultants } \\
& \text { 7. Radiors }
\end{aligned}
$$

Figure 4 Stakeholder analysis map with guiding coalition representatives highlighted.

criticism if complications occurred. Those we spoke to felt this may be due to public perception that they were less skilled or less well trained than doctors. This was tackled in the teaching session by highlighting the fact that this was felt to be a low force, non-leverage technique that was more about positioning, gravity and relaxation. As such, it could be considered a low risk technique, reinforced by the fact that none of the literature had reported any significant complications.

Following the training session, the ENPs were free to use the new equipment and technique under distant supervision from senior doctors overseeing the 'See and Manage' area of the department. They went on to use the bench autonomously once they felt comfortable to do so.

Within 2 weeks, we had three separate nurse practitioners use the bench successfully with a large amount of both patient and practitioner satisfaction. We were cautious about 'declaring victory too early', as this is referred to by Kotter as one of the key reasons why change often fails. ${ }^{13}$

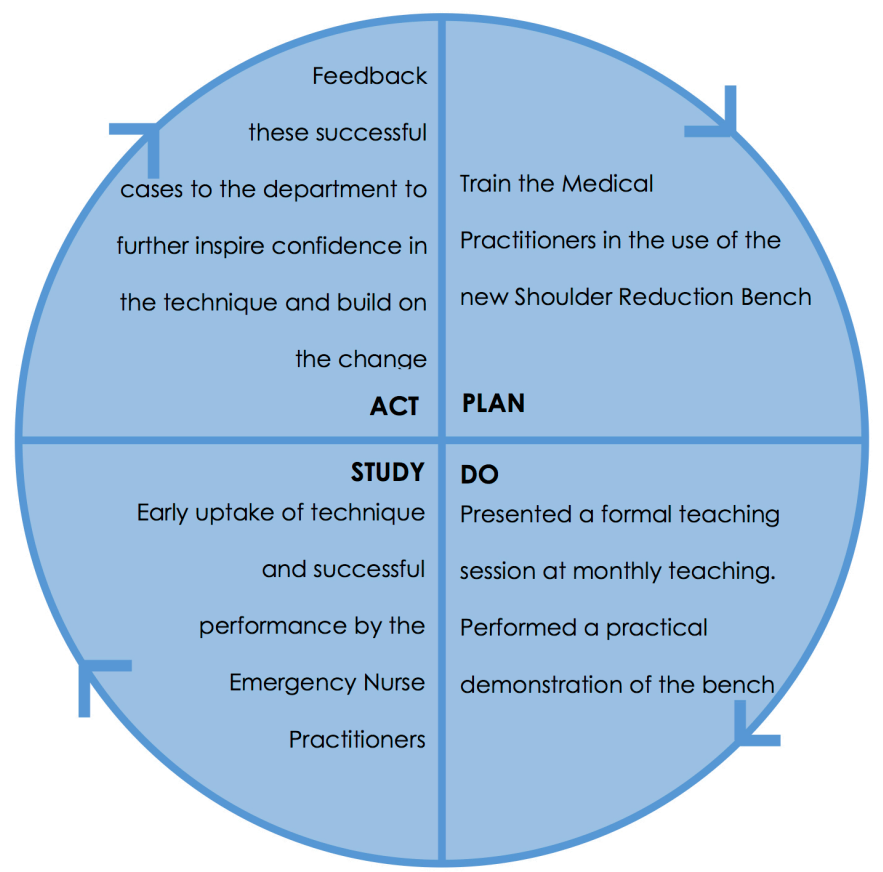

Figure 5 Plan, Do, Study, Act Cycle 1.

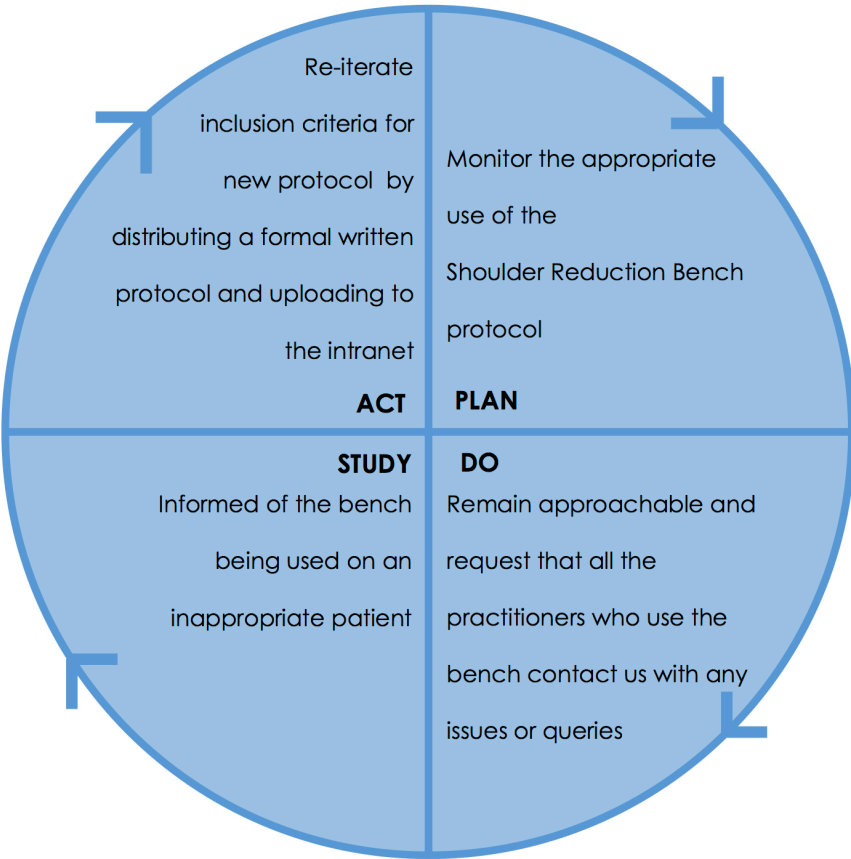

Figure 6 Plan, Do, Study, Act Cycle 2.

It was more important to use these early stories of 'quick-wins' to reinforce the change process (figure 5). We now knew the bench could be used effectively and independently by the nurse practitioners in our department. This could prove very valuable in inspiring their colleagues. A departmental email was sent to all staff informing them of these successes and thanked them for taking on the project with such enthusiasm.

There was one account of a failed reduction by a nurse practitioner on a posterior shoulder dislocation. This prompted the production of a written protocol and flowchart to communicate the inclusion and exclusion criteria for the protocol more widely (figure 6). The protocol (figure 7) was then uploaded to the emergency department intranet so that it could be easily accessed in the future and help anchor the change. This was important to do as there was a danger that the bench was being used on inappropriate patients. This could result in an increased failure rate, poor patient care and loss of confidence in the technique for the wrong reasons.

Regular one-to-one teaching sessions were not practical considering the number of rotational staff. With a practical procedure such as this, it was felt that a training video featuring a real patient would be of most value and would be more sustainable in terms of continued access to training for the staff (figure 8).

Link to training video: https://www.youtube.com/ watch?v=40aCqhfQXD4\&feature=youtu.be

\section{Measures}

The measures analysed to demonstrate any change in patient care were: 
Sunderland Royal Hospital Emergency Department Adult Shoulder Reduction Protocol

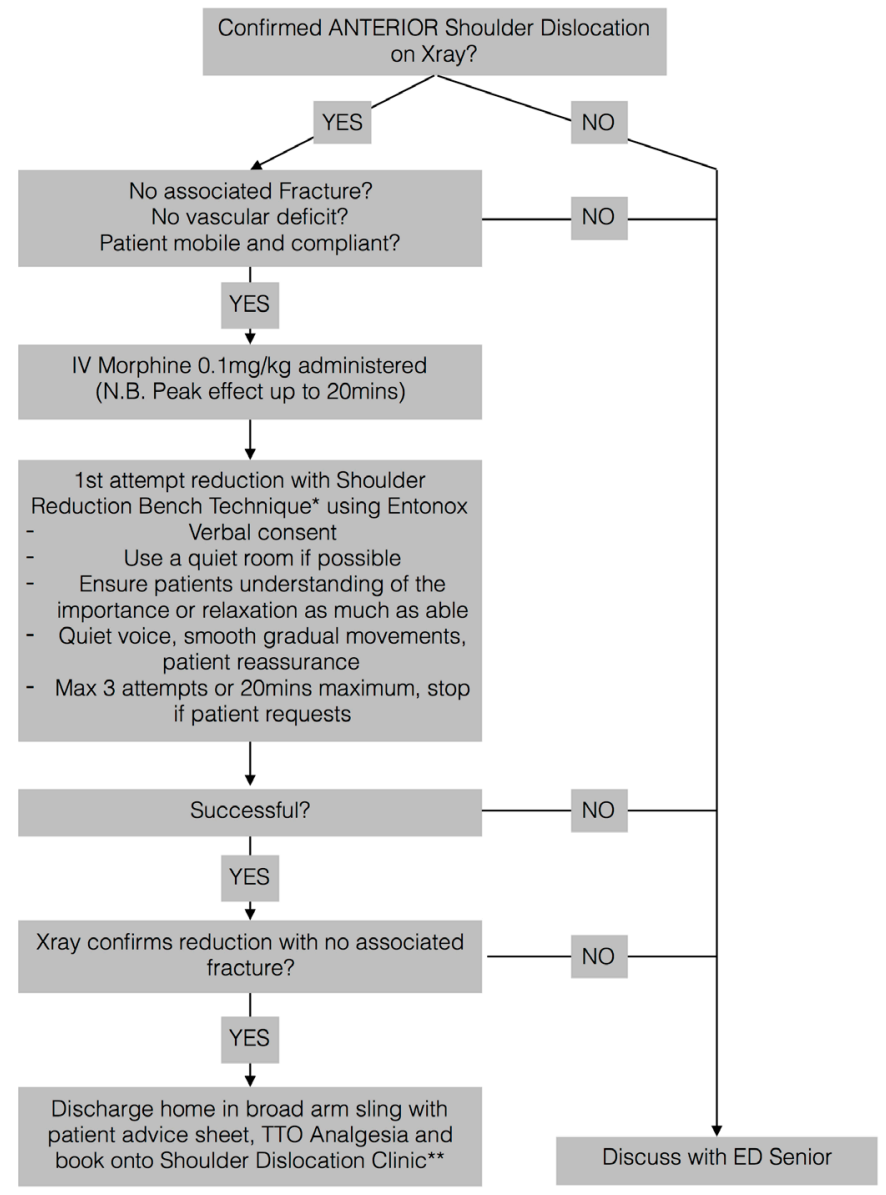

* Training video can be accessed at: https://youtu.be/40aCqhfQXD4 ** The next available Shoulder Dislocation Clinic appointment can be booked onto by the ED receptionists using the clinic code: T\&OPHY/UPLIMB. If the clinic is unavailable then book onto next day fracture clinic.

Figure 7 Written Shoulder Reduction protocol.

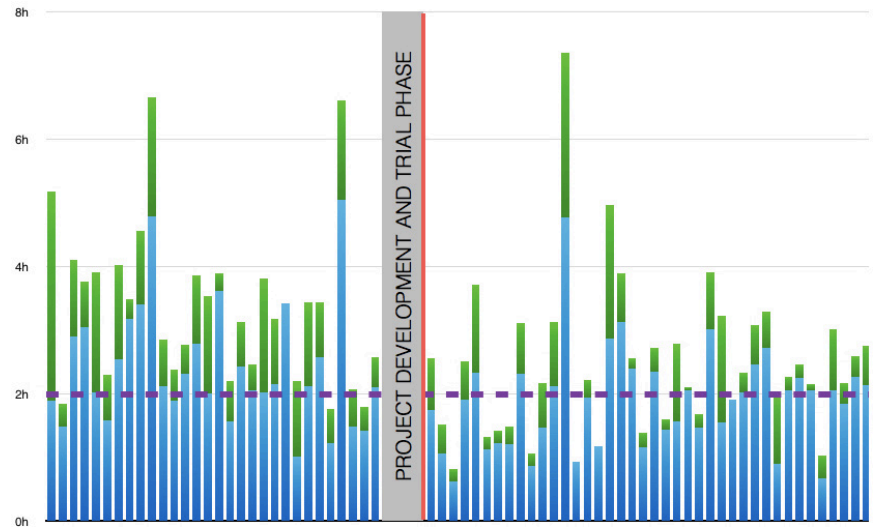

Figure 9 Run chart showing outcome measures of each case of anterior shoulder dislocation presenting pre and post the Shoulder Reduction Bench Project launch. Blue: Time from triage to confirmed shoulder relocation on X-ray. Green: Time from triage to patient discharge from the emergency department. Purple dotted line: 2-hour RCEM clinical standard of $75 \%$ initial attempt shoulder reduction. Red line: Project launch.

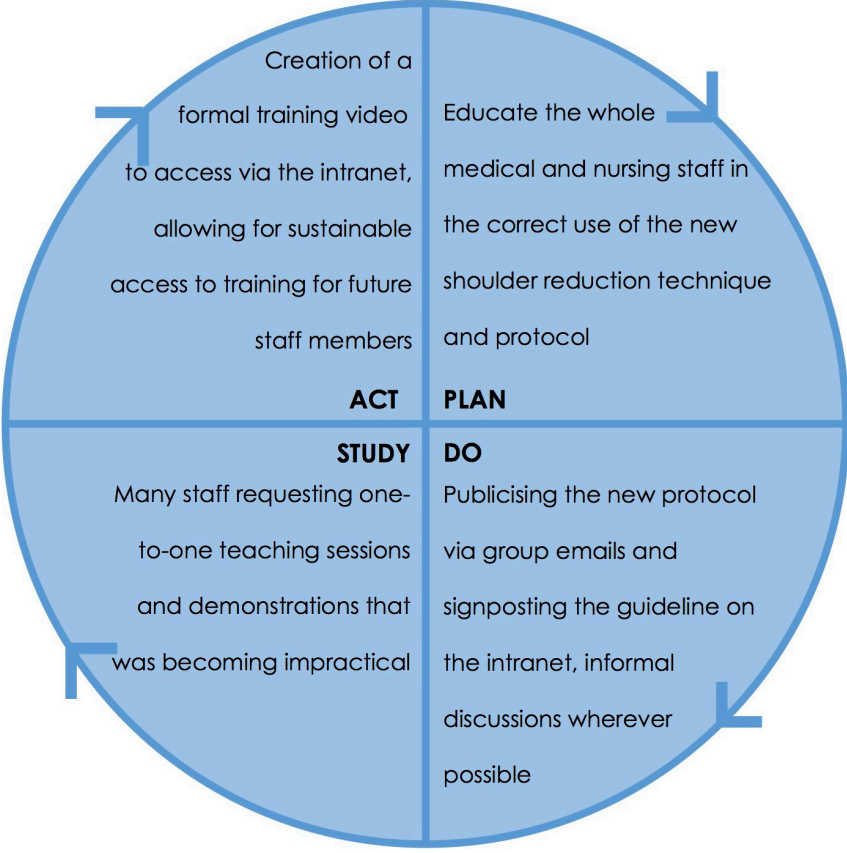

Figure 8 Plan, Do, Study, Act 3.

\section{Outcome measures}

- Time from triage to X-ray confirming shoulder reduction.

- Time from triage to patient discharge from the emergency department.

\section{Process measure}

- Proportion of patients receiving procedural sedation. Using the 'Launchpad' data collection system, we were able to identify each patient who presented to the emergency department with a diagnosis code 'Dislocation of the Shoulder Joint'. We then discounted patients who did not meet our protocol's inclusion criteria and those who required reduction in theatre. The data were collected on a monthly basis for 6 months after the launch of the project.

\section{RESULTS}

The run chart of the data collected for 6 months from the project's launch is shown in figure 9. The initial audit data are shown for comparison.

There was no delay in the uptake of the new shoulder dislocation protocol. Throughout the 6-month data collection, a total of 40 patients were identified as meeting the inclusion criteria. There were two cases $(5 \%)$ where the new shoulder dislocation protocol was not followed; on both occasions, the practitioner involved was a consultant who reduced the shoulder with a different technique with nitrous oxide only. In a further two cases (5\%), it was not clear in the documentation whether the practitioner had adhered to the protocol and these patients were treated with morphine only. The other $90 \%$ had all apparently followed the protocol as per the flowchart. Three patients $(7.5 \%)$ refused morphine and were 


\begin{tabular}{|c|c|c|c|}
\hline & Preprotocol $(n=30)$ & $\begin{array}{l}\text { Postprotocol } \\
(\mathrm{n}=40)\end{array}$ & Change \\
\hline $\begin{array}{l}\text { Average time from triage to confirmed shoulder } \\
\text { reduction on X-ray }\end{array}$ & 2 hours $25 \mathrm{~min}$ & 1 hour $54 \mathrm{~min}$ & 31 min $(21 \%)$ reduction \\
\hline $\begin{array}{l}\text { Average time from triage to patient discharge from } \\
\text { emergency department }\end{array}$ & 3 hours $22 \mathrm{~min}$ & 2 hours $30 \mathrm{~min}$ & 52 min (26\%) reduction \\
\hline Percentage of patients sedated & $57 \%(17)$ & $18 \%(7)$ & $68 \%$ reduction \\
\hline
\end{tabular}

reduced using the new technique with nitrous oxide only, and the remainder $(82.5 \%)$ received a single titrated dose of morphine and nitrous oxide for an attempted reduction with the new technique. Seven patients $(18 \%)$ were unable to be treated successfully using the bench and went on to receive procedural sedation with either Midazolam or Propofol.

The outliers preprotocol and postprotocol, with time to discharge over 6 hours, were all cases when the patient had a delayed diagnosis of a shoulder dislocation due to an inability to communicate a clear history of injury.

Table 3 shows the average results of the outcome and process measures studied. Results are given both preintroduction and 6 months postintroduction of the new shoulder reduction protocol.

Referring back to the original process map (figure 1), we can show the change in the treatment routes that patients took following the new shoulder protocol (figure 10).

Having addressed the case whereby the new technique was used on a patient who did not meet the protocol's inclusion criteria, there were no further complications or safety issues arising from the project. This is in keeping with the previous studies into use of the Oxford Chair where no complications were identified.

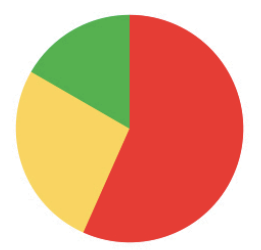

RED: SEDATION ROUTE 57\%

AMBER: HAND OVER ANALGESIA ONLY ROUTE $27 \%$

GREEN: SINGLE PRACTITIONER ANALGESIA ONLY ROUTE $16 \%$

POST-PROTOCOI

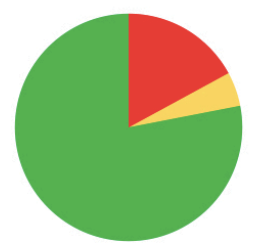

RED: SEDATION ROUTE $18 \%$

AMBER: HAND OVER ANALGESIA ONLY ROUTE 4\%

GREEN: SINGLE PRACTITIONER ANALGESIA ONLY ROUTE $78 \%$

Figure 10 Pie charts comparing what percentage of patients with shoulder dislocations were managed along each process pathway preintroduction and postintroduction of the new shoulder dislocation protocol.

\section{DISCUSSION}

\section{Summary and interpretation}

Putting this into context of the care a patient receives, as a result of our new shoulder dislocation protocol, patients are less likely to receive potentially dangerous sedating medication and on average will have their shoulders reduced and be discharged more quickly.

The success rates of the new shoulder technique would appear to correlate with the results quoted in the previous literature studying the original Oxford Chair.

A particular strength of this project has been the relative simplicity of the shoulder bench technique. In the time since the project's conception, there has been a systematic review published by Alkaduhimi $e t a l^{14}$ which included a study of the Oxford Chair. This review concluded that the scapular manipulation and 'FARES' method were the most favourable in terms of duration of stay and least pain during reduction. However, unlike these methods, the shoulder bench technique is difficult to get wrong as it carries an added benefit of positioning the patient for you, with only a small extra force required in line with gravity.

Another strength of this project is that the target population is clearly identifiable and has an obvious immediate cure for their condition and pain. This results in high patient satisfaction rates and a knock-on effect towards practitioner job satisfaction and ongoing enthusiasm.

The cost is also very low, with only a single piece of equipment required and simple 'in-house' or online training.

We had not anticipated the results of the new shoulder protocol being quite so impressive and prompt. This is thanks, in the main, to the nurse practitioners that we had targeted when educating the department. When considering the Kurt Lewin model of change, there is normally an unfreezing process required prior to change and refreezing with a new approach (figure 11). ${ }^{15}$

As most nurse practitioners had no prior experience in reducing shoulders, there was little unfreezing process required, unlike their doctor counterparts who may have their own way of managing this injury based on previous experience. This is highlighted by the fact that the only two practitioners documented to not use the protocol appropriately were consultants. Their management approaches are far more firmly set. The only unfreezing process for the nurse practitioners was to move on from 


\section{UNFREEZE $\longrightarrow$ CHANGE $\longrightarrow$ REFREEZE}

Figure 11 The Kurt Lewin model of change.

the previously held belief that such procedures were beyond their competency.

While we believe the outcomes to be sustainable, this will rely on the ENPs remaining in post and continuing to develop this skill. The initial feedback from the ENPs has been resoundingly positive regarding the additional job satisfaction that this new practical skill brings. We hope that with open access to the local training video and the recent development of Senior Band 8 ENPs tasked with ongoing training for new recruits that we will continue to keep this cohort of staff current, competent and confident in this new technique.

The real cost savings to the department as a result of the project are overall reduced bed occupancy (both general and monitored beds) and the subsequent effect that this has on ambulance handover times. It also frees up hours of senior clinician and nursing time needed to sedate patients. There is also the potential to reduce adverse events in the department due to any complications of conscious sedation and save on the investigative processes or potential litigation costs.

The sacrifice in terms of opportunity cost to the department would mostly be training and experience related. Due to the success of the protocol, the majority of our shoulder dislocations are now reduced by nurse practitioners. Doctors in training may, therefore, be exposed to fewer shoulder dislocations and sedations. These are mandatory skills for the Emergency Medicine trainee. We will endeavour to tackle this by ensuring doctors rotate around the minor injuries department to gain experience in the shoulder protocol where possible. There must also be a conscious effort to involve the junior doctors in the cases where the shoulder bench has been unsuccessful and reduction under sedation is required. This was reiterated to the nurse practitioners at our initial teaching session.

\section{Limitations}

This project's generalisability is limited by the fact that the culture of sedating such a high proportion of patients for shoulder reductions may have only been relevant to our hospital.

Our hospital has the region's highest number of nurse practitioners working alongside doctors on the junior medical rota. With their involvement being so key to the success of this project, this may also limit generalisability.

There were limitations in the data collected. Time to postreduction X-ray was selected as an indicator of the speed with which patients were treated, as these X-rays are automatically timestamped and more reliable than a successful reduction time documented in the notes. The time of procedure was rarely documented and often done in retrospect. It is accepted that the triage time to postreduction X-ray time will be longer for those patients who have been sedated as they need to recover from their medication before being transferred to X-ray without monitoring. There will also be varied waiting times for X-ray depending on how busy the radiology department is. Therefore, having a delayed X-ray time does not mean that the shoulder dislocation treatment was necessarily delayed. Assuming this is constant in the preprotocol and postprotocol data, we can still interpret the obvious trend towards improved time to X-ray and therefore extrapolate that to suggest improved time to treatment.

The data collected may be confounded by the period in which it was collected. The preprotocol audit data were collected from December to October, that is, a number of winter months. The postprotocol data were studied from March to September. The emergency department may have been significantly busier over the winter months with resources stretched. This may have impacted on patients' time to treatment and introduced bias into the results.

\section{CONCLUSION}

In addition to the benefits for patients, the Shoulder Reduction Bench project has been useful to the department in a number of ways. It has reduced the occupancy of monitored beds and reduced the strain on the senior doctors and nursing staff. It has provided a cohort of staff with a new skill which provides improved job satisfaction and may make posts more appealing when recruiting new staff in the future. Having changed the pre-existing culture that nurse practitioners could not perform shoulder reductions, there will likely be an increase in confidence and the drive to train them further to manage other common injuries; dislocated ankles, dislocated elbows, femoral shaft fractures.

These improvements align with the Royal College of Emergency Medicine's Vision 2020, which prioritises the formation of a broader clinical workforce and reorganising systems to provide better, faster and safer care. ${ }^{16}$

With a permanent staff base now trained and experienced in reducing anterior shoulder dislocations and an online training resource, these changes will likely be sustained. The durability of the bench is unknown, however. We have worked with our innovations department to help develop our prototype bench further.

For more information on the design of the Shoulder Reduction Bench, please contact: Philip.dowson@chsft. nhs.uk

Acknowledgements Dr Ala Mohammed designed and manufactured the Shoulder Reduction Bench and will act as guarantor. Dr Phil Hormbrey designed the Oxford Chair. Ms Amanda Hay collected initial audit data. Ms. Charlotte Fox assisted with data analysis. Dr Alex Johnston reviewed the paper. Thanks to the staff at Sunderland Royal Hospital Emergency Department for supporting this project.

Funding The authors have not declared a specific grant for this research from any funding agency in the public, commercial or not-for-profit sectors.

Competing interests None declared.

Patient consent for publication Not required.

Provenance and peer review Not commissioned; externally peer reviewed. 
Open access This is an open access article distributed in accordance with the Creative Commons Attribution Non Commercial (CC BY-NC 4.0) license, which permits others to distribute, remix, adapt, build upon this work non-commercially, and license their derivative works on different terms, provided the original work is properly cited, appropriate credit is given, any changes made indicated, and the use is non-commercial. See: http://creativecommons.org/licenses/by-nc/4.0/.

\section{REFERENCES}

1. Shah A, Judge A, Delmestri A, et al. Incidence of shoulder dislocations in the UK, 1995-2015: a population-based cohort study. BMJ Open 2017;7:e016112.

2. The College of emergency medicine clinical standards for emergency departments 2014.

3. Jaques K, Beard D, Dewar A, et al. Procedural sedation and analgesia in the emergency department: what are the factors associated with complications? Proceedings of the 5th European Congress on emergency medicine. Munich, Germany; 2008 September 15-18th. Eur J Emerg Med 2008;15:293-4.

4. Sacchetti A, Senula G, Strickland J, et al. Procedural sedation in the community emergency department: initial results of the ProSCED registry. Acad Emerg Med 2007;14:41-6.

5. Safe Sedation of Adults in the Emergency Department. Report and recommendations by the Royal College of anaesthetists and the College of emergency medicine Working Party on sedation. Anaesthesia and Airway Management in the Emergency Department, 2012.
6. National Quality Board. Supporting NHS providers to deliver the right staff, with the right skills, in the right place at the right time, 2016.

7. Smith SLF. An investigation comparing the Oxford chair technique with the traditional methods of glenohumeral dislocation reduction currently implemented. Int Emerg Nurs 2009;17:38-46.

8. Hormbrey P, Smith S. Shoulder dislocation and the Oxford chair. Emerg Med J 2014;31:255.3-6.

9. Wheeless Online. Reduction of anterior dislocation. Available: http:// wheelessonline.com/ortho/reduction_of_anterior_dislocation

10. Chung JYM, Cheng $\mathrm{CH}$, Graham CA, et al. The effectiveness of a specially designed shoulder chair for closed reduction of acute shoulder dislocation in the emergency department: a randomised control trial. Emerg Med J 2013;30:795-800.

11. Guler O, Ekinci S, Akyildiz F, et al. Comparison of four different reduction methods for anterior dislocation of the shoulder. J Orthop Surg Res 2015;10.

12. The National Archives. Available: https://webarchive.nationalarchives. gov.uk/20141206090408/http://www.mhra.gov.uk/Howweregulate/ Devices/Complyingwithlegislation/ActivelmplantableMedicalDevi cesDirective/Inhousemanufacture/index.htm

13. Kotter J. Leading change. Boston: Harvard Business School Press, 1996.

14. Alkamuhdi $\mathrm{H}$, van der Linde JA, Willigenburg NW, et al. A systematic comparison of the closed shoulder reduction techniques. Arch Orthop Trauma Surg 2017;137:589-99.

15. Lewin K. Frontiers in group dynamics: concept, method and reality in social science; social equilibria and social change. Human Relations 1947:34-5

16. RCEM Vision. 2020: fixing emergency department staffing, systems and support to deliver excellent patient centred care, 2017. 